TRANSACTIONS OF THE

AMERICAN MATHEMATICAL SOCIETY

Volume 228, 1977

\title{
EXAMPLES FOR THE NONUNIQUENESS OF THE EQUILIBRIUM STATE(1)
}

BY

FRANZ HOFBAUER

\begin{abstract}
In this paper equilibrium states on shift spaces are considered. A uniqueness theorem for equilibrium states is proved. Then we study a particular class of continuous functions. We characterize the functions of this class which satisfy Ruelle's Perron-Frobenius condition, those which admit a measure determined by a homogeneity condition, and those which have unique equilibrium state. In particular, we get examples for the nonuniqueness of the equilibrium state.
\end{abstract}

0. Introduction. In [7] Walters proved a very general variational principle for dynamical systems. He considered a compact metric space $X$ and a continuous map $T: X \rightarrow X . C(X)$ denotes the Banach space of all real-valued continuous functions on $X$. The pressure is defined as real-valued function $P$ on $C(X)$ (see $\S 1$ of [7]). Walters showed that $P(\varphi)=\sup _{\mu \in M_{T}(X)}\left(h_{\mu}(T)+\mu(\varphi)\right)$, where $M_{T}(X)$ denotes the collection of all $T$-invariant probability measures on $X$ and $h_{\mu}(T)$ the entropy of $\mu$ with respect to $T$. A $\mu$ such that $h_{\mu}(T)+\mu(\varphi)$ attains its supremum is called equilibrium state. Walters based himself on a paper by Ruelle. [6], who considered a $Z^{n}$ action on a compact metric space and proved a variational principle, when the action is expansive and satisfies the specification condition.

Intrinsically ergodic dynamical systems, i.e. those having a unique measure maximizing entropy, have been thoroughly investigated (see e.g. [9]). A more general question is to find dynamical systems $(X, T)$ and functions $\varphi \in C(X)$ admitting a unique equilibrium state.

Bowen [1] considers one-sided subshifts $\Sigma_{A}^{+}$of finite type and the set $\mathscr{F}_{A} \subset C\left(\Sigma_{A}^{+}\right)$of those functions which are Hölder continuous with respect to a certain metric on $\Sigma_{A}^{+}$. For each $\varphi \in C\left(\Sigma_{A}^{+}\right)$he defines the operator $\mathfrak{L}_{\varphi}$ on $C\left(\Sigma_{A}^{+}\right)$by $\mathcal{L}_{\varphi} f(\underline{x})=\sum_{y \in \sigma^{-1} \underline{x}} e^{\varphi(\underline{y})} f(\underline{y})$ and shows that, if $\varphi \in \mathscr{F}_{A}, \mathcal{L}_{\varphi}$ satisfies the Ruelle-Perron-Frobenius (RPF) condition, i.e. there are $\lambda>0, h$ $\in C\left(\Sigma_{A}^{+}\right)$with $h>0$ and $\nu \in M\left(\Sigma_{A}^{+}\right)$, the set of all Borel probability

Received by the editors December 8, 1975.

AMS (MOS) subject classifications (1970). Primary 28A65; Secondary 47A35, 54H20.

(1) This article is part of a Ph.D. thesis written at the University of Vienna. 
measures, for which $\mathcal{L}_{\varphi} h=\lambda h, \mathcal{L}_{\varphi}^{*} \nu=\lambda \nu, \nu(h)=1$ and $\lambda^{-m} \mathcal{L}_{\varphi}^{m} f$ converges uniformly to $\nu(f) h$ for every $f \in C\left(\Sigma_{A}^{+}\right)$[1, Theorem 1.7]. By this condition $\lambda, \nu$ and $h$ are uniquely determined. Furthermore $\lambda=e^{P(\varphi)}$ and the RPFmeasure $\mu$ defined by $\mu(f)=\nu(h f)$ is shift invariant.

From the above properties of $\nu$ and $h$ Bowen deduces for $\varphi \in \mathscr{F}_{A}$ that $\mu$ satisfies a homogeneity condition, i.e. there are $c_{1}, c_{2}>0$ and $P$ such that

$$
c_{1} \leqslant \frac{\mu\left({ }_{0}\left[x_{0} x_{1} \cdots x_{m-1}\right]\right)}{\exp \left(-P m+\sum_{k=0}^{m-1} \varphi\left(\sigma^{k} \underline{x}\right)\right)} \leqslant c_{2}
$$

for every $\underline{x} \in \Sigma_{A}^{+}$and every $m>0$ [1, Theorem 1.16]. By this condition $P$ and $\mu$ are uniquely determined and $P=P(\varphi)$. The measure $\mu$ will be called homogeneous.

Bowen then shows that the homogeneous measure is an equilibrium state for $\varphi \in \mathscr{F}_{A}$ and that this equilibrium state is unique [1, Theorem 1.22].

The purpose of this paper is to consider these problems for functions which are not Hölder continuous. In $\$ 1$ we bring the definitions and prove that every continuous function for which the RPF-condition is valid has a unique equilibrium state. This follows immediately from the methods deduced in the papers of Walters [8] and Ledrappier [5] and generalizes Bowen's result in [1].

The remainder of the paper deals with a particular class of functions, which were studied earlier in statistical mechanics. The corresponding situation there is called the Fisher model (see [4] and [2]). In $\$ 2$ of this paper we characterize the functions of this class which satisfy the RPF-condition, in $\$ 3$ those which admit a homogeneous measure, and in $\$ \S 4$ and 5 those which have unique equilibrium state. In particular, $\$ 4$ gives examples for the nonuniqueness of the equilibrium state. $\$ 5$ also contains a summary of these results.

1. Let $\Sigma_{A}^{+}$denote a subshift of $\Sigma_{n}^{+}=\Pi_{0}^{\infty}\{1,2, \ldots, n\}$ of finite type and $\sigma$ the one-sided shift on it. $M\left(\Sigma_{A}^{+}\right)$denotes the collection of all Borel probability measures of $\Sigma_{A}^{+}$and $M_{\sigma}\left(\Sigma_{A}^{+}\right)$the collection of all $\sigma$-invariant ones. For each $\varphi \in C\left(\Sigma_{A}^{+}\right)$, the Banach space of all real-valued continuous functions with supremum norm $\|\cdot\|$, we define the operator $\mathcal{L}_{p}$ on $C\left(\Sigma_{A}^{+}\right)$by

$$
\mathcal{E}_{\varphi} f(\underline{x})=\sum_{y \in \sigma^{-1} \underline{x}} e^{\varphi(\underline{y})} f(\underline{y})
$$

Set $S_{m} \varphi(\underline{x})=\sum_{i=0}^{m-1} \varphi\left(\sigma^{i} \underline{x}\right)$. Then

$$
\mathcal{L}_{\varphi}^{m} f(\underline{x})=\sum_{y \in \sigma^{-m} \underline{x}} \exp \left(S_{m} \varphi(\underline{y})\right) f(\underline{y}) .
$$

We shall say that $\varphi$ satisfies the RPF-condition if there are $\lambda>0, h$ $\in C\left(\Sigma_{A}^{+}\right)$with $h>0$ and $\nu \in M\left(\Sigma_{A}^{+}\right)$for which $\mathcal{L}_{\varphi} h=\lambda h, \mathcal{L}_{\varphi}^{*} \nu=\lambda \nu, \nu(h)$ 
$=1$ and $\left\|\lambda^{-m} \mathfrak{L}_{\varphi}^{m} f-\nu(f) h\right\| \rightarrow 0$ for all $f \in C\left(\Sigma_{A}^{+}\right)$. The measure $\mu$ defined by $\mu(f)=\nu(h f)$ will be called RPF-measure. Define the pressure $P: C\left(\Sigma_{A}^{+}\right)$ $\rightarrow R \cup\{+\infty\}$ by

$$
P(\varphi)=\lim \frac{1}{m} \log \sum_{x_{0}} \sum_{x_{m-1}} \sup \exp S_{m} \varphi(\underline{y}),
$$

where the supremum is taken over all $y \in{ }_{0}\left[x_{0} \cdots x_{m-1}\right]=\left\{\underline{y \in \Sigma_{A}^{+}: y_{i}}\right.$ $\left.=x_{i}, 0 \leqslant i \leqslant m-1\right\}$. Then $\mu \in M_{\sigma}\left(\Sigma_{A}^{+}\right)$is called an equilibrium state for $\varphi$ if $P(\varphi)=h_{\mu}+\mu(\varphi)$, where $h_{\mu}$ denotes the entropy of $\mu$ relative to $\sigma$ and $\mu(\varphi)=\int \varphi d \mu$.

As an easy consequence of Walters' paper [8] we have

(1.1) THEOREM. Let $\varphi$ satisfy the RPF-condition. Then $\mu=\nu$ is the unique equilibrium state for $\varphi$.

Proof. Set $\bar{\varphi}=\varphi+\log h-\log h \circ \sigma-\log \lambda$. By Lemma 1.1 of [8] $\varphi$ and $\bar{\varphi}$ have the same equilibrium states. We write $\mathcal{L}$ for $\mathfrak{L}_{\varphi}$ and $\overline{\mathcal{L}}$ for $\mathfrak{L}_{\bar{\phi}} . \bar{\varphi}$ satisfies the RPF-condition with $\bar{\nu}=\mu, \bar{h}=1$ and $\bar{\lambda}=1$ as the following calculation shows:

$$
\overline{\mathrm{L}} 1(\underline{x})=\sum_{y \in \sigma^{-1} \underline{x}} e^{\varphi(\underline{y})} \frac{1}{\lambda} h(\underline{y}) \frac{1}{h(\underline{x})}=\frac{1}{\lambda} \frac{1}{h(\underline{x})} \operatorname{L} h(\underline{x})=1 .
$$

Hence $\bar{\complement} 1=1$.

$$
\begin{aligned}
\overline{\mathfrak{L}}^{*} \mu(f) & =\mu(\overline{\mathfrak{L}} f)=\nu\left(h \lambda^{-1} h^{-1} \mathfrak{L} h f\right)=\lambda^{-1} \nu(\mathcal{L} h f) \\
& =\nu(h f)=\mu(f) .
\end{aligned}
$$

Hence $\overline{\mathfrak{L}}^{*} \mu=\mu$.

$$
\begin{aligned}
\overline{\mathfrak{L}}^{m} f & =\sum_{y \in \sigma^{-m_{\underline{x}}}} e^{S_{m} \varphi(\underline{y})} \frac{1}{\lambda^{m}} h(\underline{y}) \frac{1}{h\left(\sigma^{m} \underline{y}\right)} f(\underline{y})=\frac{1}{\lambda^{m}} \frac{1}{h} \mathfrak{L}^{m} h f \\
& \rightarrow h^{-1} \nu(h f) h=\mu(f) .
\end{aligned}
$$

Hence $\overline{\mathfrak{L}}^{m} f \rightarrow \mu(f)$ uniformly.

$\bar{\phi}$ satisfies the requirement $\sum_{y \in \sigma^{-1} \underline{x}} e^{\bar{\phi}(\underline{y})}=1$. Therefore, for $m \in M\left(\Sigma_{A}^{+}\right)$, the following are equivalent (see Theorem 2.1 of [8])

(i) $\overline{\mathrm{L}}^{*} m=m$.

(ii) $m \in M_{\sigma}\left(\Sigma_{A}^{+}\right)$and $m$ is an equilibrium state for $\bar{\phi}$.

Now if $\mu$ is the RPF-measure $\nu$, we have $\overline{\mathfrak{L}}^{*} \mu=\mu$. Hence $\mu$ is an equilibrium state for $\bar{\varphi}$ and, hence, for $\varphi$.

On the other hand, if $\mu$ is an equilibrium state for $\varphi$, it is one for $\bar{\phi}$, hence $\overline{\mathfrak{E}}^{*} \mu=\mu$. As $\bar{\varphi}$ satisfies the RPF-condition and the RPF-measure is uniquely 
determined by this condition, the theorem is proved.

2. Throughout the remainder of this paper we shall deal with particular functions, which we define now. Set $M_{0}=\Sigma_{n}^{+} \backslash_{0}[1]$ and $M_{k}=\left\{\underline{x} \in \Sigma_{n}^{+}: x_{i}=1\right.$ for $\left.0 \leqslant i \leqslant k-1, x_{k} \neq 1\right\}$ for $k=1,2, \ldots$. Then the sets $M_{k}$ together with the point $\{11 \cdots\}$ form a partition of $\Sigma_{n}^{+}$. Let $\left(a_{k}\right)$ be a sequence of real numbers with $\lim a_{k}=0$. Set $s_{k}=a_{0}+\cdots+a_{k}$. Define $g \in C\left(\Sigma_{n}^{+}\right)$by

$$
g(\underline{x})=a_{k} \text { for } \underline{x} \in M_{k} \text { and } g(11 \cdots)=0 \text {. }
$$

THEOREM. If $\sum_{k=0}^{\infty} e^{s_{k}}>1 /(n-1)$, then $g$ satisfies the RPF-condition.

Proof. The existence of $\lambda>0$ and $\nu \in M\left(\Sigma_{n}^{+}\right)$follows from the Schauder-Tychonoff theorem as in the proof of Theorem 1.7 of [1]. To calculate $\nu$ define $\nu_{k}=\nu\left(M_{k}\right)$ and $\nu_{\infty}=\nu(11 \cdots)$. $\mathcal{L}^{*} \nu=\lambda \nu$ implies

$$
\lambda \nu_{0}=\lambda \nu\left(M_{0}\right)=\nu\left(\left[1_{M_{0}}\right)=\nu\left(\sum_{i=1}^{n} e^{g(i \underline{x})} 1_{M_{0}}(i \underline{x})\right)=(n-1) e^{a_{0}}\right.
$$

and

$$
\begin{aligned}
\lambda \nu_{k} & =\lambda \nu\left(M_{k}\right)=\nu\left(\varrho 1_{M_{k}}\right)=\nu\left(\sum_{i=1}^{n} e^{g(i \underline{x})} 1_{M_{k}}(\underline{i} \underline{x})\right) \\
& =\nu\left(e^{g(\underline{1} \underline{x})} 1_{M_{k-1}}(\underline{x})\right)=e^{a_{k}} \nu_{k-1} .
\end{aligned}
$$

From (2.2) and (2.3) it follows that

$$
\nu_{k}=\lambda^{-k-1}(n-1) e^{s_{k}} .
$$

$\left(M_{k}\right)_{k=0}^{\infty}$ together with the point $11 \cdots$ form a partition of $\Sigma_{n}^{+}$, hence

$$
\begin{aligned}
\lambda\left(\sum_{k=0}^{\infty} \nu_{k}+\nu_{\infty}\right) & =\lambda \nu(1)=\nu(\mathcal{L} 1)=\nu\left(\sum_{i=1}^{n} e^{g(i \underline{x})}\right) \\
& =\nu\left(e^{g(\underline{1} \underline{x})}\right)+(n-1) e^{a_{0}} \\
& =\nu\left(\sum_{k=1}^{\infty} e^{a_{k}} 1_{M_{k-1}}\right)+e^{0} \nu_{\infty}+(n-1) e^{a_{0}} \\
& =\sum_{k=1}^{\infty} \lambda \nu_{k}+\lambda \nu_{0}+\nu_{\infty} \quad \text { by (2.2) and (2.3). }
\end{aligned}
$$

From this one gets

(2.5) $(\lambda-1) \nu_{\infty}=0$.

(2.6) $\lambda>1$, because $\lambda \leqslant 1$ implies by (2.4) 


$$
1=\sum_{k=0}^{\infty} \nu_{k}+\nu_{\infty} \geqslant(n-1) \sum_{k=0}^{\infty} e^{s_{k}}+\nu_{\infty}>1
$$

Thus it follows from (2.5) that $\nu_{\infty}=0$.

To calculate $h$ define $A_{k}(\underline{x})=\mathfrak{L}^{k} 1(\underline{x})$ for $k \geqslant 1$ and $A_{0}(\underline{x})=1$. Then for $k \geqslant 1$,

$$
\begin{aligned}
A_{k}(\underline{x}) & =\sum_{y_{0} \cdots y_{k-1}} e^{S_{k} g\left(y_{0} y_{1} \cdots y_{k-1} \underline{x}\right)} \\
& =\sum_{y_{k-1}=1}^{n} e^{g\left(y_{k-1} \underline{x}\right)} \sum_{y_{k-2}=1}^{n} \cdots \sum_{y_{0}=1}^{n} e^{g\left(y_{0} \cdots y_{k-1} \underline{x}\right)} .
\end{aligned}
$$

Now

$$
\sum_{y_{0}=1}^{n} e^{g\left(y_{0} \cdots y_{k-1} \underline{x}\right)}=e^{g\left(1 y_{1} \cdots y_{k-1} \underline{x}\right)}+(n-1) e^{a_{0}}
$$

and, hence,

$$
\begin{aligned}
A_{k}(\underline{x})= & \sum_{y_{k-1}} \exp \left\{g\left(y_{k-1} \underline{x}\right)\right\} \sum_{y_{k-2}} \cdots \\
& \sum_{y_{1}} \exp \left\{g\left(y_{1} \cdots y_{k-1} \underline{x}\right)+g\left(1 y_{1} \cdots y_{k-1} \underline{x}\right)\right\} \\
& +(n-1) e^{a_{0}} A_{k-1}(\underline{x}) .
\end{aligned}
$$

Again

$$
\begin{aligned}
\sum_{y_{1}} \exp \left\{g\left(y_{1} \cdots y_{k-1} \underline{x}\right)+g\left(1 y_{1} \cdots y_{k-1} \underline{x}\right)\right\} & \\
& =\exp \left\{g\left(1 y_{2} \cdots y_{k-1} \underline{x}\right)+g\left(11 y_{2} \cdots y_{k-1} \underline{x}\right)\right\}+(n-1) e^{s_{1}} A_{k-2}(\underline{x}) .
\end{aligned}
$$

Iterating this step one gets

$$
\begin{aligned}
A_{k}(\underline{x})= & \sum_{y_{k-1}} \exp \left\{g\left(y_{k-1} \underline{x}\right)\right\} \cdots \\
& \sum_{y_{m}} \exp \left\{g\left(y_{m} \cdots y_{k-1} \underline{x}\right)+\cdots+g\left(1 \cdots 1 y_{m} \cdots y_{k-1} \underline{x}\right)\right\} \\
& +(n-1) e^{s_{m-1}} A_{k-m}(\underline{x})+\cdots+(n-1) e^{s_{0}} A_{k-1}(\underline{x}),
\end{aligned}
$$

and therefore,

$$
\begin{aligned}
A_{k}(\underline{x})= & e^{g(\underline{1} \underline{x})+\cdots+g(1 \cdots \underline{1})}+(n-1) e^{s_{k-1}} A_{0}(\underline{x})+\cdots \\
& +(n-1) e^{s_{0}} A_{k-1}(\underline{x}) .
\end{aligned}
$$

Define $B_{k}(\underline{x})=\lambda^{-k} A_{k}(\underline{x})$. Choose $t$ such that $\underline{x} \in M_{t}$. Then an easy calculation using (2.4) shows 


$$
\begin{aligned}
& B_{k}(\underline{x})=\nu_{t}^{-1} \nu_{t+k}+\nu_{k-1} B_{0}(\underline{x})+\cdots+\nu_{0} B_{k-1}(\underline{x}) \text { for } \underline{x} \neq 11 \cdots, \\
& B_{k}(11 \cdots)=\lambda^{-k}+\nu_{k-1} B_{0}(11 \cdots)+\cdots+\nu_{0} B_{k-1}(11 \cdots) .
\end{aligned}
$$

Define $u_{i}$ by

$$
u_{0}=1 \text { and } u_{i}=u_{i-1} \nu_{0}+\cdots+u_{0} \nu_{i-1} \text {. }
$$

Then

$$
\begin{aligned}
u_{0} B_{k}(\underline{x})+\cdots+ & u_{k} B_{0}(\underline{x}) \\
& =u_{0}\left(\nu_{0} B_{k-1}(\underline{x})+\cdots+\nu_{k-1} B_{0}(\underline{x})+\nu_{t}^{-1} \nu_{t+k}\right)+\cdots+u_{k} .
\end{aligned}
$$

Rearranging terms and using (2.10) one gets

$$
\begin{aligned}
& B_{k}(\underline{x})=\nu_{t}^{-1}\left(u_{0} \nu_{t+k}+u_{1} \nu_{t+k-1}+\cdots+u_{k} \nu_{t}\right) \text { for } \underline{x} \neq 11 \cdots, \\
& B_{k}(11 \cdots)=u_{0} \lambda^{-k}+\cdots+u_{k-1} \lambda+u_{k} .
\end{aligned}
$$

By the Renewal Theorem (see [3, p. 306]),

$$
\lim u_{k}=\left(\sum_{j=1}^{\infty} j v_{j-1}\right)^{-1} \stackrel{:}{=} u
$$

because the $\nu_{i}$ satisfy $\nu_{i}>0$ and $\sum_{i=0}^{\infty} \nu_{i}=1$.

Now we show that $B_{k}(\underline{x})$ converges uniformly to $h \in C\left(\Sigma_{n}^{+}\right)$defined by

$$
\begin{aligned}
& h(\underline{x})=u v_{t}^{-1} \sum_{i=t}^{\infty} \nu_{i} \stackrel{:}{=} h_{t} \text { for } \underline{x} \in M_{t}, \\
& h(11 \cdots)=u \sum_{i=0}^{\infty} \lambda^{-i} .
\end{aligned}
$$

For $\underline{x} \neq 11 \cdots$ one has, using $(2.11)$,

$$
\begin{aligned}
\mid B_{k}(\underline{x})- & u v_{t}^{-1} \sum_{i=t}^{\infty} \nu_{i} \mid \\
& \leqslant \nu_{t}^{-1}\left(\nu_{t}\left|u_{k}-u\right|+\cdots+\nu_{t+k}\left|u_{u}-u\right|+\nu_{t+k+1} u+\cdots\right) .
\end{aligned}
$$

Fix $\varepsilon>0$. As $\sum \nu_{i}$ is convergent, there is a $K$ with $\sum_{i=K+1}^{\infty} \nu_{t+i}<\varepsilon$. Choose $M$ so that $\left|u_{k-K}-u\right|<\varepsilon$ for every $k \geqslant M$. Then

$$
\begin{aligned}
\left|B_{k}(\underline{x})-u \nu_{t}^{-1} \sum_{i=t}^{\infty} \nu_{i}\right| & \leqslant \nu_{t}^{-1}\left(\sum_{i=0}^{K} \nu_{t+i}\left|u_{k-i}-u\right|+C \sum_{i=K+1}^{\infty} \nu_{t+i}\right) \\
& \leqslant \nu_{t}^{-1}(\varepsilon+C \varepsilon) \text { for every } k \geqslant M,
\end{aligned}
$$


where $C=\max \left(u, \sup \left|u_{i}-u\right|\right)$. Therefore $B_{k}(\underline{x}) \rightarrow u v_{t}^{-1} \sum_{i=t}^{\infty} \nu_{i}$ uniformly on $M_{t}$ for every $t$. Similarly for $\underline{x}=11 \cdots: B_{k}(11 \cdots) \rightarrow u \sum_{i=0}^{\infty} \lambda^{-i}$.

To show that the convergence is uniform, choose $N$ in the following way: By (2.6) there is a $c>0$ with $e^{c}<\lambda$. Since $a_{n} \rightarrow 0$ there exists $N$ with $\left|a_{n}\right| \leqslant c$ for every $n \geqslant N$. Setting $q=\lambda^{-1} e^{c}<1$, one obtains for $t \geqslant N$,

$$
\nu_{t}^{-1} \nu_{t+i}=\lambda^{-i} e^{a_{t+i}+\cdots+a_{t+1}} \leqslant \lambda^{-i} e^{c i}=q^{i} .
$$

Fix $\varepsilon>0$. There is a constant $K$ (independent of $t$ ) such that

$$
\sum_{i=K+1}^{\infty} \nu_{t}^{-1} \nu_{t+i} \leqslant \sum_{i=K+1}^{\infty} q^{i}=q^{K+1}(1-q)^{-1}<\varepsilon \text { for every } t \geqslant N .
$$

Choose $M$ as above. Then

$$
\begin{aligned}
\left|B_{k}(\underline{x})-u \nu_{t}^{-1} \sum_{i=t}^{\infty} \nu_{i}\right| & \leqslant \sum_{i=0}^{K} \nu_{t}^{-1} \nu_{t+i}\left|u_{k-i}-u\right|+C \sum_{i=K+1}^{\infty} \nu_{t}^{-1} \nu_{t+i} \\
& \leqslant(1-q)^{-1} \varepsilon+C \varepsilon \text { for every } k \geqslant M \text { and every } t \geqslant N .
\end{aligned}
$$

Hence

$$
B_{k}(\underline{x})=\lambda^{-k} \mathfrak{L}^{k} 1(\underline{x}) \quad \text { converges uniformly to the function } h
$$

defined by (2.12), which therefore must be continuous.

Furthermore, $h>0$, because $h_{t}=u\left(\nu_{t} \nu_{t}^{-1}+\cdots\right) \geqslant u>0$. $\mathscr{L}$ is a bounded operator on $C\left(\Sigma_{n}^{+}\right)\left(\|\mathcal{I}\| \leqslant n e^{\|\ell\|}\right)_{t}$. Hence,

$$
\lambda^{-1} \mathfrak{L} h=\lambda^{-1} \mathfrak{L} \lim \lambda^{-k} \mathfrak{L}^{k} 1=\lim \lambda^{-k-1} \mathfrak{L}^{k+1} 1=h
$$

or

$$
\mathfrak{L} h=\lambda h .
$$

Now it remains to show $\lambda^{-k} \mathfrak{L}^{k} f \rightarrow \nu(f) h$ uniformly for every $f \in C\left(\Sigma_{n}^{+}\right)$. By the proof of Theorem 1.7 of [1] it suffices to show this for $f \in C_{m}$ (i.e. for functions which are constant on the cylinders of the form $\left.0\left[x_{0} \cdots x_{m-1}\right]\right)$ and by the linearity of $\mathcal{L}$ and $\nu$ for $f=1_{\left.d x_{0} \cdots x_{m-1}\right]}$. Then

Choose $r$ such that $x_{r-1} \neq 1$ and $x_{r}=\cdots=x_{m-1}=1(0 \leqslant r \leqslant m)$. 


$$
\begin{aligned}
\lambda^{-r} \mathcal{E}^{r} 1_{d}\left[x_{0} \cdots x_{m-1}\right] & (z) \\
& =\lambda^{-r} \sum_{y_{0} \cdots y_{r-1}} e^{S_{r} g\left(y_{0} \cdots y_{r-1} z\right)} 1_{d}\left[x_{0} \cdots x_{m-1}\right] \\
& =\lambda^{-r} e^{S_{r} g\left(x_{0} \cdots x_{r-1} z\right)} 1_{d\left[x_{0} \cdots x_{m-1}\right]}\left(x_{0} \cdots y_{r-1} \underline{z}\right) \\
& =\text { constant } \cdot 1_{d\left[x_{r} \cdots x_{m-1}\right]}(z)=\text { constant } \cdot 1_{d}[11 \cdots 1]
\end{aligned}
$$

$\left(S_{r} g\left(x_{0} \cdots x_{r-1} z\right)\right.$ is independent of $\underline{z}$, because $x_{r-1} \neq 1$.) Hence it suffices to consider $1_{0[11 \cdots 1]}\left(x_{0}=x_{1}=\cdots=x_{m-1}=1\right)$. For $k>m$ one has

$$
\begin{aligned}
\lambda^{-k} \underline{L}^{k} 1_{d}[11 \cdots 1] & (\underline{z}) \\
& =\lambda^{-k} \sum_{y_{0} \cdots y_{k-1}} e^{S_{k} g\left(y_{0} \cdots y_{k-1} \underline{z}\right)} 1_{d[11 \cdots 1]}\left(y_{0} \cdots y_{k-1} \underline{z}\right) \\
& =\lambda^{-k} \sum_{y_{m} \cdots y_{k-1}} e^{S_{k} g\left(11 \cdots 1 y_{m} \cdots y_{k-1} \underline{z}\right)} \\
& =\lambda^{-k}\left(A_{k}(\underline{z})-(n-1) e^{s_{0}} A_{k-1}(\underline{z})-\cdots-(n-1) e^{s_{m-1}} A_{k-m}(\underline{z})\right) \\
& =B_{k}(\underline{z})-\left(\nu_{0} B_{k-1}(\underline{z})+\cdots+\nu_{m-1} B_{k-m}(\underline{z})\right) \\
& \rightarrow h(\underline{z})-\left(\nu_{0} h(\underline{z})+\cdots+\nu_{m-1} h(\underline{z})\right)=\nu\left(1_{d}[11 \cdots 1]\right) h(\underline{z}) .
\end{aligned}
$$

By (2.13) the convergence is uniform and the theorem is proved.

REMARK. If $\sum_{k=0}^{\infty} e^{s_{k}} \leqslant 1 /(n-1)$, then $g$ does not satisfy the RPFcondition, because $\lambda=1$ by $(2.5)$ and therefore $B_{k}(11 \cdots)$ tends to $+\infty$.

REMARK. The theorem is also valid for subshifts $\Sigma_{A}^{+}$of $\Sigma_{n}^{+}$of finite type, if the first row of $A$ consists only of 1 and each column of $A$ contains the same number of 1's, say $m$. Then the requirement $\sum_{k=0}^{\infty} e^{s_{k}}>1 /(n-1)$ must be replaced by $\sum_{k=0}^{\infty} e^{s_{k}}>1 /(m-1)$.

3. $g$ is said to admit a homogeneous measure $\mu \in M_{\sigma}\left(\Sigma_{n}^{+}\right)$, if there are $c_{1}, c_{2}>0$ and $\lambda>0$ such that

$$
c_{1} \leqslant \frac{\mu\left({ }_{0}\left[x_{0} x_{1} \cdots x_{m-1}\right]\right.}{\lambda^{-m} \exp S_{m} g(\underline{x})}=Q \leqslant c_{2}
$$

for every $\underline{x} \in \Sigma_{n}^{+}$and every $m \geqslant 0$.

THEOREM. The function $g$ defined by (2.1) has a homogeneous measure iff $\sum_{k=0}^{\infty} a_{k}$ is convergent. 
PRoof. First we shall show that, if $\sum a_{k}$ is divergent, there is no homogeneous measure.

Suppose there is one. Then by (3.1) one has for $\underline{y}, \underline{z} \in{ }_{0}\left[x_{0} \cdots x_{m-1}\right]$,

$$
c_{1} \exp S_{m} g(\underline{y}) \leqslant \lambda^{m} \mu\left({ }_{0}\left[x_{0} \cdots x_{m-1}\right]\right) \leqslant c_{2} \exp S_{m} g(\underline{z}) .
$$

Taking the logarithm and setting $K=\log c_{2}-\log c_{1}, S_{m} g(\underline{y}) \leqslant S_{m} g(\underline{z})+K$ and, therefore (change the places of $\underline{y}$ and $\underline{z}$ ),

$$
\left|S_{m} g(\underline{y})-S_{m} g(\underline{z})\right| \leqslant K .
$$

Now choose $x_{0}=\cdots=x_{m-1}=1, y_{i}=2$ for $i \geqslant m$ and $z_{i}=1$ for $i \geqslant m$. Then (3.2) becomes $\left|a_{1}+a_{2}+\cdots+a_{m}\right| \leqslant K$, a contradiction.

If $\sum a_{k}$ is convergent, the RPF-condition is satisfied. We shall show that $\mu=\nu h$ is a homogeneous measure. For the $\sigma$-invariance of $\mu$ see [1, p. 21].

(3.3) Since there are $d_{1}, d_{2}>0$ with $d_{1} \leqslant h \leqslant d_{2}$, it suffices to check the homogeneous condition (3.1) for $\nu$ instead of $\mu$. Set $\bar{Q}$ $=\nu\left({ }_{0}\left[x_{0} \cdots x_{m-1}\right]\right) /\left(\lambda^{-m} \exp S_{m} g(\underline{x})\right)$. Choose $t$ so that $\sigma^{m} \underline{x} \in M_{t}$ and $r$ the smallest integer between 0 and $m$ such that $x_{r}=\cdots=x_{m-1}=1$. Then

$$
\begin{aligned}
\nu\left({ }_{0}\left[x_{0} \cdots x_{m-1}\right]\right) & =\lambda^{-r} \nu\left(\mathscr{L}^{r} 1_{0}\left[x_{0} \cdots x_{m-1}\right]\right) \\
& =\lambda^{-r} \exp \left(S_{r} g\left(x_{0} \cdots x_{r-1} \cdots\right)\right) \nu\left(1_{0\left[x_{0} \cdots x_{m-1}\right]}\right) \\
& =\lambda^{-r} \exp \left(S_{r} g\left(x_{0} \cdots x_{r-1} \cdots\right)\right) \sum_{i=m-r}^{\infty} v_{i} .
\end{aligned}
$$

$\left(S_{r} g\left(x_{0} \cdots x_{r-1} \cdots\right)\right.$ depends only on $x_{0} \cdots x_{r-1}$, because $x_{r-1} \neq 1$.)

$$
\begin{aligned}
S_{m} g(\underline{x})= & S_{r} g\left(x_{0} \cdots x_{r-1} \cdots\right) \\
& +g\left(x_{r} \cdots x_{m-1} \cdots\right)+\cdots+g\left(x_{m-1} \cdots\right) \\
= & S_{r} g\left(x_{0} \cdots x_{r-1} \cdots\right)+a_{m-r+t}+\cdots+a_{1+t} .
\end{aligned}
$$

From (3.4) and (3.5) one gets

$$
\bar{Q}=\lambda^{m-r} e^{-s_{m-r+l}+s_{t}} \sum_{i=m-r}^{\infty} \nu_{i}
$$

$\sum a_{k}$ is convergent, hence there is a constant $K$ with $\left|s_{k}\right| \leqslant K$ for every $k$. This implies

$$
e^{-2 K} \leqslant e^{-s_{m-r+t}+s_{t}} \leqslant e^{2 K}
$$

Furthermore, 


$$
\begin{aligned}
\lambda^{m-r} \sum_{i=m-r}^{\infty} \nu_{i} & =(n-1) \sum_{i=0}^{\infty} \lambda^{-i-1} e^{s_{m-r+i}} \text { by (2.4) } \\
& =(n-1) e^{s_{m-r}} \sum_{i=0}^{\infty} \lambda^{-i-1} e^{s_{m-r+i}-s_{m-r}} \\
& \leqslant(n-1) e^{K} \sum_{i=0}^{\infty} \lambda^{-i-1} e^{b_{i}}
\end{aligned}
$$

where $b_{i}=\sup _{j \geqslant 0}\left|s_{j+i}-s_{j}\right| \cdot a_{k} \rightarrow 0$ implies $i^{-1} b_{i} \rightarrow 0$ and, therefore, the convergence radius of the series $\sum e^{b_{i}} x^{i+1}$ is 1 . Hence $\sum \lambda^{-i-1} e^{b_{i}}$ is convergent. Set $(n-1) e^{K} \sum \lambda^{-i-1} e^{b_{i}}=L$.

On the other hand it is clear that

$$
\lambda^{m-r} \sum_{i=m-r}^{\infty} \nu_{i} \geqslant(n-1) \lambda^{-1} e^{s_{m-r}}=M>0
$$

Hence

$$
M \leqslant \lambda^{m-r} \sum_{i=m-r}^{\infty} \nu_{i} \leqslant L
$$

By (3.6), (3.7) and (3.8) one gets $M e^{-2 K} \leqslant \bar{Q} \leqslant L e^{2 K}$ and the proof is completed by (3.3).

Remark that, if $\sum a_{k}$ is divergent, condition (3.1) may be "almost" satisfied (in the sense of (3.12) and (3.15) below) for the measure $\mu=\nu h$.

For example choose $a_{k}=\log (k+2) /(k+1)$. Then $s_{k}=\log (k+2)$ and $v_{k}=(n-1)(k+2) \lambda^{-k-1}$. We have $s_{k} \rightarrow \infty$, but by (3.6),

$$
\begin{aligned}
\bar{Q} & =\lambda^{m-r} e^{-s_{m-r+t}+s_{t}} \sum_{i=m-r}^{\infty} v_{i} \\
& =\lambda^{m-r} \frac{t+2}{m-r+t+2}(n-1) \sum_{i=m-r}^{\infty}(i+2) \lambda^{-i-1} .
\end{aligned}
$$

For $|x|<1$ one has

$$
\sum_{i=k}^{\infty}(i+2) x^{i+1}=\frac{(k+2) x^{k+1}-(k+1) x^{k+2}}{(1-x)^{2}} .
$$

Using this for $x=\lambda^{-1}<1$ and $k=m-r$,

$$
\begin{aligned}
\bar{Q} & =(n-1) \frac{t+2}{m-r+t+2} \frac{(m-r+2) \lambda-(m-r+1)}{(\lambda-1)^{2}} \\
& \geqslant \frac{2(n-1)}{(\lambda-1)^{2}} \frac{(m-r+2) \lambda-(m-r+1)}{m-r+2} \geqslant \frac{2(n-1)}{(\lambda-1)^{2}}(\lambda-1) \\
& =2(n-1) /(\lambda-1)=\bar{c}_{1}>0 .
\end{aligned}
$$


Hence by (3.3) the constant $c_{1}$ of (3.1) exists. As $\sum \log (k+2) /(k+1)$ is divergent, $c_{2}$ cannot exist by the above result.

But one can even show more: Set

$$
Q_{\text {sup }}=\frac{\mu\left({ }_{0}\left[x_{0} \cdots x_{m-1}\right]\right)}{\lambda^{-m} \sup \exp S_{m} g(y)},
$$

where the supremum is taken over all $\underline{y} \in{ }_{0}\left[x_{0} \cdots x_{m-1}\right]$, and analogously $\bar{Q}_{\text {sup }}$. As $S_{m} g(y)$ attains this supremum for $y=x_{0} \cdots x_{m-1} 22 \cdots$ (this means $t=0)$ one has, by (3.9),

$$
\begin{aligned}
\bar{Q}_{\text {sup }} & =(n-1) \frac{2}{m-r+2} \frac{(m-r+2) \lambda-(m-r+1)}{(\lambda-1)^{2}} \\
& =\frac{2(n-1)}{(\lambda-1)^{2}}\left(\lambda-\frac{m-r+1}{m-r+2}\right) \leqslant \frac{2(n-1)}{(\lambda-1)^{2}}\left(\lambda-\frac{1}{2}\right)=\bar{c}_{2} .
\end{aligned}
$$

By (3.3), (3.9) and (3.11) there are $c_{1}, c_{2}>0$ such that

$$
c_{1} \leqslant Q_{\text {sup }} \leqslant c_{2} \text { and } c_{1} \leqslant Q \text {. }
$$

For another example choose $a_{k}=-\log (k+2) /(k+1)$. Then

$$
s_{k}=-\log (k+2) \text { and } \nu_{k}=(n-1)(k+2)^{-1} \lambda^{-k-1} \text {. }
$$

Again $s_{k} \rightarrow-\infty$. By (3.6)

$$
\begin{aligned}
\bar{Q} & =\lambda^{m-r} e^{-s_{m-r+t}+s_{t}} \sum_{i=m-r}^{\infty} v_{i} \\
& =\lambda^{m-r} \frac{m-r+t+2}{t+2}(n-1) \sum_{i=m-r}^{\infty} \frac{1}{i+2} \lambda^{-i-1} .
\end{aligned}
$$

Now $(m-r+t+2) /(t+2)(i+2) \leqslant 1$ for every $i \geqslant m-r$, hence

$$
\bar{Q} \leqslant(n-1) \sum_{i=1}^{\infty} \lambda^{-i}=\bar{c}_{2} .
$$

By (3.3) the constant $c_{2}$ of (3.1) exists, so there cannot be a $c_{1}>0$, because $\sum-\log (k+2) /(k+1)$ is divergent.

Define $\bar{Q}_{\text {inf }}$ and $Q_{\text {inf }}$ analogously to (3.9). $S_{m} g(\underline{y})$ attains the infimum for $y=x_{0} \cdots x_{m-1} 22 \cdots($ this means $t=0)$. Hence

$$
\bar{Q}_{\text {inf }}=\frac{n-1}{2} \sum_{i=1}^{\infty} \frac{m-r+2}{m-r+i+1} \lambda^{-i} \geqslant \frac{n-1}{2} \sum_{i=1}^{\infty} \frac{2}{i+1} \lambda^{-i}=\bar{c}_{1}>0
$$

By (3.3), (3.13) and (3.14) there are $c_{1}, c_{2}>0$ such that 


$$
c_{1} \leqslant Q_{\text {inf }} \leqslant c_{2} \text { and } Q \leqslant c_{2} .
$$

4. The functions $g$ defined by (2.1) give us also examples for the nonuniqueness of the equilibrium state. Throughout this section we shall consider $\Sigma_{2}^{+}$.

(4.1) Lemma. If $\sum_{k=0}^{\infty} e^{s_{k}} \leqslant 1$ then $P(g) \leqslant 0$.

Proof. By definition $P(g)=\lim k^{-1} \log Z_{k}(g)$, where

$$
Z_{k}(g)=\sum_{x_{0} \cdots x_{k-1}} \exp \sup S_{k} g(y)
$$

the supremum taken over all $\underline{y} \in{ }_{0}\left[x_{0} \cdots x_{k-1}\right]$,

$$
\begin{aligned}
Z_{k}(g)= & \sum_{r=1}^{k} \sum_{x_{0}} \cdots x_{r-2} \exp \sup S_{k} g\left(x_{0} \cdots x_{r-2} 211 \cdots 1 y_{k} \cdots\right) \\
& +\exp \sup S_{k} g\left(11 \cdots 1 y_{k} \cdots\right) .
\end{aligned}
$$

(For each cylinder ${ }_{0}\left[x_{0} \cdots x_{k-1}\right] \neq{ }_{0}[11 \cdots 1]$ choose $r$ so that $x_{r-1}=2$ and $x_{r}=\cdots=x_{k-1}=1$.)

$$
\begin{aligned}
\sup S_{k} g\left(x_{0} \cdots x_{r-2}\right. & \left.211 \cdots 1 y_{k} y_{k+1} \cdots\right) \\
& =S_{r} g\left(x_{0} \cdots x_{r-2} 2 \cdots\right)+\sup S_{k-r} g\left(11 \cdots 1 y_{k} y_{k+1} \cdots\right) \\
& =S_{r} g\left(x_{0} \cdots x_{r-2} 2 \cdots\right)+S_{k-r} g\left(\underline{y}_{r}\right),
\end{aligned}
$$

where $\underline{y}_{r} \in{ }_{0}[11 \cdots 1]$ is chosen such that $S_{k-r} g\left(11 \cdots 1 y_{k} \cdots\right)$ attains its supremum for $y_{r} .\left(S_{r} g\left(x_{0} \cdots x_{r-2} 2 \cdots\right)\right)$ is independent of the continuation of the block $x_{0} \cdots x_{r-2} 2$.) Therefore,

$$
\begin{aligned}
& Z_{k}(g)=\sum_{r=1}^{k} \exp S_{k-r} g\left(\underline{y}_{r}\right) \sum_{x_{0}} \cdots x_{r-2} \exp S_{r} g\left(x_{0} \cdots x_{r-2} 2 \cdots\right)+\exp S_{k} g\left(y_{0}\right), \\
& \sum_{x_{0}} \cdots x_{r-2} \exp S_{r} g\left(x_{0} \cdots x_{r-2} 2 \cdots\right)=e^{a_{0}} \sum_{x_{0} \cdots x_{r-2}} \exp S_{r-1} g\left(x_{0} \cdots x_{r-2} 2 \cdots\right) \\
& =e^{a_{0}} A_{r-1}(22 \cdots) \quad(r \geqslant 1) \text { by (2.7). }
\end{aligned}
$$

Hence,

$$
Z_{k}(g)=\sum_{r=1}^{k} \exp S_{k-r} g\left(\underline{y}_{r}\right) \cdot e^{a_{0}} A_{r-1}(22 \cdots)+\exp S_{k} g\left(\underline{y}_{0}\right) .
$$

(2.9) becomes

$$
A_{k}(22 \cdots)=e^{s_{k}-s_{0}}+e^{s_{k-1}} A_{0}(22 \cdots)+\cdots+e^{s_{0}} A_{k-1}(22 \cdots) .
$$

From this one sees by induction that 


$$
A_{k}(22 \cdots) \leqslant e^{-s_{0}} \text {, hence } e^{a_{0}} A_{k}(22 \cdots) \leqslant 1 \text {. }
$$

(For $A_{0}(22 \cdots)=1$ this is clear, because $\Sigma e^{s_{k}} \leqslant 1$, therefore $e^{s_{0}} \leqslant 1$. If $A_{i}(22 \cdots) \leqslant e^{-s_{0}}$ for $i=1,2, \ldots, k-1$ then

$$
A_{k}(22 \cdots) \leqslant e^{-s_{0}}\left(e^{s_{k}}+\cdots+e^{s_{0}}\right) \leqslant e^{-s_{0}},
$$

because $\Sigma e^{s_{k}} \leqslant 1$.) Furthermore choose $t$ such that $\sigma^{k-r} \underline{y}_{r} \in M_{t}$. Then

$$
S_{k-r} g\left(\underline{y}_{r}\right)=a_{k-r+t}+\cdots+a_{1+t} \leqslant\left|a_{k+t}\right|+\cdots+\left|a_{1+t}\right|
$$

$$
\leqslant \sup _{j \geqslant 0} \sum_{i=1}^{k}\left|a_{i+j}\right|=C_{k}
$$

$$
\lim k^{-1} C_{k}=0, \text { because } a_{k} \rightarrow 0 .
$$

By (4.2), (4.3) and (4.4),

$$
Z_{k}(g) \leqslant(k+1) e^{C_{k}} .
$$

This implies

$$
\begin{aligned}
P(g) & =\lim k^{-1} \log Z_{k}(g) \\
& \leqslant \lim k^{-1} \log (k+1)+\lim k^{-1} C_{k}=0 \text { by (4.5). }
\end{aligned}
$$

Hence the lemma is proved.

(4.6) Lemma. If $\sum_{k=0}^{\infty} e^{s_{k}} \leqslant 1$ then $\delta_{11} \ldots$ is an equilibrium state for $g$.

Proof. Clearly $h_{\delta_{11} \ldots}=0$ and $\delta_{11} \ldots(g)=0$. Thus

$$
P(g) \leqslant 0=h_{\delta_{11} \ldots}+\delta_{11 \ldots}(g) .
$$

On the other hand

$$
P(g) \geqslant h_{\mu}+\mu(g) \text { for every } \mu \in M_{\sigma}\left(\Sigma_{2}^{+}\right)
$$

(see [1, p. 31]). Hence $P(g)=h_{\delta_{11} \ldots}+\delta_{11} \ldots(g)$ and the lemma is proved.

(4.8) Let $\varphi, \varphi_{n} \in C\left(\Sigma_{2}^{+}\right)$with $\varphi_{n} \rightarrow \varphi$ and let $\mu_{n}$ be an equilibrium state for $\varphi_{n}$. As $M_{\sigma}\left(\Sigma_{2}^{+}\right)$is compact and metric in the weak*-topology, there is a convergent subsequence $\left(\mu_{n_{i}}\right)$ of $\left(\mu_{n}\right)$.

LEMMA. $\mu=\lim \mu_{n_{i}}$ is an equilibrium state for $\varphi$.

Proof (Walters [7]). From $\left|P\left(\psi_{1}\right)-P\left(\psi_{2}\right)\right| \leqslant\left\|\psi_{1}-\psi_{2}\right\|[7$, Theorem 2.1(v)] one gets 


$$
\begin{aligned}
P(\varphi) & =\lim P\left(\varphi_{n}\right)=\lim \left(h_{\mu_{n}}+\mu_{n}\left(\varphi_{n}\right)\right) \\
& \leqslant \lim \left(h_{\mu_{n}}+\mu_{n}(\varphi)\right)+\lim \left\|\varphi_{n}-\varphi\right\| \\
& =\lim h_{\mu_{n_{i}}}+\lim \mu_{n_{i}}(\varphi) \\
& \leqslant h_{\mu}+\mu(\varphi) .
\end{aligned}
$$

$\left(\lim h_{\mu_{n^{\prime}}} \leqslant h_{\mu}\right.$ because $\mu \mapsto h_{\mu}$ is upper semicontinuous [1, p. 64].) By (4.7) we have $P(\varphi)=h_{\mu}+\mu(\varphi)$ and the lemma is proved.

The following lemma is well known.

(4.9) LeMMA. Let $\mu_{n} \in M_{\sigma}\left(\Sigma_{2}^{+}\right)$and suppose $\lim \mu_{n}\left({ }_{0}\left[x_{0} \cdots x_{m-1}\right]\right)$ exists for every cylinder ${ }_{0}\left[x_{0} \cdots x_{m-1}\right]$. Then $\mu_{n}$ converges in the weak*-topology to $\mu \in M_{\sigma}\left(\Sigma_{2}^{+}\right)$, which is uniquely determined by

$$
\mu\left({ }_{0}\left[x_{0} \cdots x_{m-1}\right]\right)=\lim \mu_{n}\left({ }_{0}\left[x_{0} \cdots x_{m-1}\right]\right) .
$$

Now we can prove the desired result.

THEOREM. Let $g$ defined by (2.1) satisfy

$$
\sum_{k=0}^{\infty} e^{s_{k}}=1 \text { and } \sum_{k=0}^{\infty}(k+1) e^{s_{k}}=u^{-1}<\infty
$$

Then $g$ has two equilibrium states.

Proof. For a given block $x_{0} \cdots x_{m-1} \neq 11 \cdots 1$ choose $t$ such that $x_{0}$ $=\cdots=x_{t-1}=1$ and $x_{t}=2$ and $r$ such that $x_{r-1}=2$ and $x_{r}=\cdots$ $=x_{m-1}=1$. We shall show that the measure $\mu$ defined by

$$
\mu\left({ }_{0}\left[x_{0} \cdots x_{m-1}\right]\right)=h_{t} \exp S_{r} g\left(x_{0} \cdots x_{r-2} 2 \cdots\right) \sum_{i=m-r}^{\infty} \dot{e}^{s_{i}}
$$

$$
\text { for }{ }_{0}\left[x_{0} \cdots x_{m-1}\right] \neq{ }_{0}[11 \cdots 1]
$$

$$
\mu\left({ }_{0}[11 \cdots 1]\right)=u \sum_{i=m}^{\infty}(i-m+1) e^{s_{i}},
$$

where $h_{t}=u e^{-s_{t}} \sum_{i=t}^{\infty} e^{s_{i}}$, is an equilibrium state for $g$.

To use (4.8) define $g_{n}$ by

$$
\begin{aligned}
& g_{n}(\underline{x})=g(\underline{x}) \quad \text { for } \underline{x} \notin M_{0}, \\
& g_{n}(\underline{x})=a_{0}^{(n)} \text { for } \underline{x} \in M_{0}, \text { where } a_{0}^{(n)} \searrow a_{0} .
\end{aligned}
$$

Then $\sum_{k=0}^{\infty} \exp s_{k}^{(n)}>1$, where $s_{k}^{(n)}=a_{0}^{(n)}+a_{1}+\cdots+a_{k}$. Hence $g_{n}$ satisfies the RPF-condition. By (1.1) $\mu^{n}=\nu^{n} h^{n}$ is an equilibrium state for $g_{n}$, where $\nu^{n}$ 
and $h^{n}$ are as in the RPF-condition for $g_{n}$. One has (use (3.4) and the fact that $h^{n}$ is constant on $\left.{ }_{0}\left[x_{0} \cdots x_{m-1}\right] \neq{ }_{0}[11 \cdots 1]\right)$,

$$
\begin{aligned}
& \mu^{n}\left({ }_{0}\left[x_{0} \cdots x_{m-1}\right]\right)=h_{l}^{n} \lambda_{n}^{-r} \exp S_{r} g_{n}\left(x_{0} \cdots x_{r-2} 2 \cdots\right) \sum_{i=m-r}^{\infty} \nu_{i}^{n} \\
& \text { for }{ }_{0}\left[x_{0} \cdots x_{m-1}\right] \neq{ }_{0}[11 \cdots 1], \\
& \mu^{n}\left({ }_{0}[11 \cdots 1]\right)=u^{(n)} \sum_{i=m}^{\infty}(i-m+1) \nu_{i}^{n},
\end{aligned}
$$

where $h_{t}^{n}$ is as in (2.12).

By (4.9) it suffices to show $\mu^{n}\left({ }_{0}\left[x_{0} \cdots x_{m-1}\right]\right) \rightarrow \mu\left({ }_{0}\left[x_{0} \cdots x_{m-1}\right]\right)$. This follows from (a)-(f) below (confer (4.11) and (4.13)).

(a) Define

$$
\begin{aligned}
& f(x)=\sum_{i=0}^{\infty} e^{s_{i}} x^{i+1} \text { and } \\
& f_{n}(x)=\sum_{i=0}^{\infty} \exp _{i}^{(n)} x^{i+1}=\exp \left(a_{0}^{(n)}-a_{0}\right) f(x) .
\end{aligned}
$$

Then $f$ and $f_{n}$ are strictly increasing on $[0,1], f(1)=1$ and $\lambda_{n}$ is uniquely determined by $f_{n}\left(\lambda_{n}^{-1}\right)=1$.

$f_{n} \geqslant f_{n+1}$ because $a_{0}^{(n)} \geqslant a_{0}^{(n+1)}$. This implies $\lambda_{n} \geqslant \lambda_{n+1} \geqslant 1$. Fix $c<1$. Then there is an $N$ with $f_{n}(c)=\exp \left(a_{0}^{(n)}-a_{0}\right) f(c)<1$ for every $n \geqslant N$ (as $f(c)<1$ choose $N$ such that $\left|a_{0}^{(n)}-a_{0}\right|<\log (1 / f(c))$ for every $\left.n \geqslant N\right)$. Therefore since $f_{n}$ increases, $\lambda_{n} \leqslant c^{-1}$ for every $n \geqslant N$. This means $\lambda_{n}$ $\rightarrow 1(n \rightarrow \infty)$.

$$
\begin{aligned}
& \nu_{i}^{n}=\lambda_{n}^{-i-1} \exp s_{i}^{(n)}=\lambda_{n}^{-i-1} \exp \left(a_{0}^{(n)}-a_{0}\right) e^{s_{i}} \rightarrow e^{s_{i}} \\
& \sum_{i=t}^{\infty} \nu_{i}^{n}=\exp \left(a_{0}^{(n)}-a_{0}\right) \sum_{i=t}^{\infty} e^{s_{i}} \lambda_{n}^{-i-1} \rightarrow \sum_{i=t}^{\infty} e^{s_{i}}
\end{aligned}
$$

by Abel's theorem on power series.

$$
\begin{aligned}
\sum_{i=m}^{\infty}(i-m+1) \nu_{i}^{n} & =\exp \left(a_{0}^{(n)}-a_{0}\right) \sum_{i=m}^{\infty}(i-m+1) e^{s_{i}} \lambda_{n}^{-i-1} \\
& \rightarrow \sum_{i=m}^{\infty}(i-m+1) e^{s_{i}}
\end{aligned}
$$

by Abel's theorem on power series. In particular,

$$
\frac{1}{u^{(n)}}=\sum_{i=0}^{\infty}(i+1) \nu_{i}^{n} \rightarrow \sum_{i=0}^{\infty}(i+1) e^{s_{i}}=\frac{1}{u} .
$$

Hence $u^{(n)} \rightarrow u$. 
(e)

$$
h_{t}^{n}=u^{(n)}\left(\nu_{t}^{n}\right)^{-1} \sum_{i=t}^{\infty} \nu_{i}^{n} \rightarrow u e^{-s_{t}} \sum_{i=t}^{\infty} e^{s_{i}}=h_{t}
$$

by (b), (c) and (d).

$$
\begin{aligned}
S_{r} g_{n}\left(x_{0} \cdots x_{r-2} 2 \cdots\right) & =l\left(a_{0}^{(n)}-a_{0}\right)+S_{r} g\left(x_{0} \cdots x_{r-2} 2 \cdots\right) \\
\rightarrow & S_{r} g\left(x_{0} \cdots x_{r-2} 2 \cdots\right)
\end{aligned}
$$

where $l$ is the number of 2's in the block $x_{0} \cdots x_{r-2} 2$.

We have proved that the measure $\mu$ defined by (4.11) is an equilibrium state for each $g$ satisfying (4.10). But as for this $g, \sum e^{s_{k}}=1, \delta_{11} \ldots$ is also an equilibrium state by (4.6) and $\mu \neq \delta_{11} \ldots$ because $\mu(\{11 \cdots\})=0$. Hence the theorem is proved.

It remains to show that there is a sequence $\left(a_{k}\right)$ of real numbers satisfying

(a) $a_{k} \rightarrow 0$.

(b) $\sum_{k=0}^{\infty} e^{s_{k}}=1$.

(c) $\sum_{k=0}^{\infty}(k+1) e^{s_{k}}<\infty$.

Proof. Choose

$$
a_{k}=-3 \log ((k+2) /(k+1)) \text { for } k \geqslant 1
$$

and

$$
a_{0}=-\log \left(1+\sum_{k=1}^{\infty} e^{a_{1}+\cdots+a_{k}}\right)
$$

Then

(a) $\quad \log ((k+2) /(k+1)) \rightarrow 0$.

(b)

$$
\sum_{k=0}^{\infty} e^{s_{k}}=e^{a_{0}}\left(1+\sum_{k=1}^{\infty} e^{a_{1}+\cdots+a_{k}}\right)=1 \text {. }
$$

(c)

$$
\sum_{k=0}^{\infty}(k+1) e^{s_{k}}=e^{a_{0}}+\sum_{k=1}^{\infty}(k+1)(k+2)^{-3}<\infty .
$$

5. In this section we give a summary of the results about the functions defined by (2.1) in the form of the following table and complete the proofs of these results. 


\begin{tabular}{|c|c|c|c|c|}
\hline & & $\begin{array}{l}g \text { satisfies } \\
\text { the RPF- } \\
\text { condition }\end{array}$ & $\begin{array}{c}g \text { admits a } \\
\text { homogeneous } \\
\text { measure }\end{array}$ & $\begin{array}{c}g \text { has unique } \\
\text { equilibrium } \\
\text { state }\end{array}$ \\
\hline \multirow{2}{*}{$\sum e^{s} k>\frac{1}{n-1}$} & $\sum a_{k}$ converges & yes & yes & yes \\
\hline & $\sum a_{k}$ diverges & yes & no & yes \\
\hline \multirow{2}{*}{$\sum e^{s} k=\frac{1}{n-1}$} & $\sum(k+1) e^{s k}<\infty$ & no & no & no \\
\hline & $\sum(k+1) e^{s} k=\infty$ & no & no & yes \\
\hline$\sum e^{s} k<\frac{1}{n-1}$ & & no & no & yes \\
\hline
\end{tabular}

The first column of this tible has been proved in $\S 2$, the second one in $\S 3$. The first both "yes" in the last column follow from the first both "yes" in the first column by (1.1). The "no" has been proved in $\S 4$ for $\Sigma_{2}^{+}$, but it is easily carried over to the case of $\Sigma_{n}^{+}$.

It remains for this section to prove the last both "yes" in the third column. Again we give the proofs for $\Sigma_{2}^{+}$.

THEOREM. If $\sum e^{s_{k}}<1$, then $\delta_{11} \ldots$ is the unique equilibrium state for $g$.

Proof. Suppose $g$ has an equilibrium state $\mu \neq \delta_{11} \ldots$. Then there is an $M_{i}$ with $\mu\left(M_{i}\right)>0$. Define $\bar{g}$ by

$$
\begin{array}{ll}
\bar{g}(\underline{x})=g(\underline{x}) & \text { for } \underline{x} \notin M_{i}, \\
\bar{g}(\underline{x})=\bar{a}_{i} & \text { for } \underline{x} \in M_{i}
\end{array}
$$

with $\bar{a}_{i}>a_{i}$ such that $\sum e^{\bar{s}_{k}}<1$, where $\bar{s}_{k}=a_{0}+\cdots+\bar{a}_{i}+\cdots+a_{k}$. Then one has $P(\bar{g})=0=P(g)=h_{\mu}+\mu(g)<h_{\mu}+\mu(\bar{g})$, a contradiction to (4.7).

For the other case we need a lemma.

(5.2) LeMMA. Let $b$ with $0<b<1$ and $\varepsilon$ with $0<\varepsilon<1-b$ be given. Then for every $\delta>0$ there is an $x$ with $0<x<\delta$ such that

$$
\frac{x}{x+\log (1-b)-\log \left(1-b e^{x}\right)}>1-b-\varepsilon .
$$

Proof. For $0<x<\log b^{-1},(5.3)$ is equivalent to

$$
\left(1-b e^{x}\right) \exp ((b+\varepsilon) /(1-b-\varepsilon)) x>1-b .
$$


Setting $f(x)=\left(1-b e^{x}\right) \exp ((b+\varepsilon) /(1-b-\varepsilon)) x$ it suffices to prove $f(x)$ $>f(0)$ for an $x \in] 0, \min \left(\delta, \log b^{-1}\right)\left[\right.$. Now one has $f^{\prime}(0)=\varepsilon /(1-b-\varepsilon)$ $>0$. The continuity of $f^{\prime}$ implies that there is an $\left.x \in\right] 0, \min \left(\delta, \log b^{-1}\right)[$ with $f^{\prime}(\xi)>0$ for all $\left.\xi \in\right] 0, x[$. Hence, by the mean value theorem, $f(x)-f(0)$ $=f^{\prime}(\xi) x>0$.

THEOREM. If $\sum e^{s_{k}}=1$ and $\sum(k+1) e^{s_{k}}=\infty$, then $\delta_{11} \ldots$ is the unique equilibrium state for $\mathrm{g}$.

Proof. Suppose $g$ has an equilibrium state $\mu \neq \delta_{11} \ldots$. Set $\mu_{i}=\mu\left(M_{i}\right)$ for $i \geqslant 0$. Our goal is to prove

$$
\mu_{0}>0 \text { and }
$$$$
\mu_{i} \geqslant \mu_{0} \sum_{k=i}^{\infty} e^{s_{k}} \text { for every } i \text {. }
$$

For both proofs we shall need $\bar{g} \in C\left(\Sigma_{2}^{+}\right)$defined for every $i$ by

$$
\begin{array}{ll}
\bar{g}(\underline{x})=g(\underline{x}) & \text { for } \underline{x} \notin M_{0} \cup M_{i}, \\
\bar{g}(\underline{x})=\bar{a}_{0} & \text { for } \underline{x} \in M_{0}, \\
\bar{g}(\underline{x})=\bar{a}_{i} & \text { for } \underline{x} \in M_{i}
\end{array}
$$

with $\bar{a}_{i}$ and $\bar{a}_{0}$ such that $\sum e^{\bar{s}_{k}}=1$, where $\bar{s}_{k}=\bar{a}_{0}+\cdots+a_{k}(k \leqslant i-1)$ and $s_{k}=a_{0}+\cdots+a_{i}+\cdots+a_{k}(k \geqslant i)$.

To prove (5.4) suppose $\mu_{0}=0$. Because of $\mu \neq \delta_{11} \ldots$ there must be an $M_{i}$ with $\mu\left(M_{i}\right)>0$. Choose in (5.6) $\bar{a}_{i}>a_{i}$ and $\bar{a}_{0}<a_{0}$. Then $P(\bar{g})=0$ $=P(g)=h_{\mu}+\mu(g)<h_{\mu}+\mu(\bar{g})$, a contradiction to (4.7). Hence (5.4) is valid.

To prove (5.5) choose in (5.6) $\bar{a}_{i}<a_{i}$ and $\bar{a}_{0}>a_{0}$. By the definition of $\bar{s}_{k}$ one has

$$
\exp \left(\bar{a}_{i}-a_{i}\right) \sum_{k=i}^{\infty} e^{s_{k}}=\exp \left(a_{0}-\bar{a}_{0}\right) \sum_{k=i}^{\infty} e^{s_{k}}
$$

Because of $\mu(\bar{g}) \leqslant P(\bar{g})-h_{\mu}=P(g)-h_{\mu}=\mu(g)$ one has

$$
\mu_{i} \bar{a}_{i}+\mu_{0} \bar{a}_{0} \leqslant \mu_{i} a_{i}+\mu_{0} a_{0} .
$$

(5.7) and (5.8) imply

$$
\frac{\mu_{i}}{\mu_{0}} \geqslant \frac{x}{x+\log (1-b)-\log \left(1-b e^{x}\right)}
$$

where $x=\bar{a}_{0}-a_{0}$ and $b=\sum_{k=0}^{i-1} e^{s_{k}}\left(\right.$ then $\left.1-b e^{x}=\sum_{k=i}^{\infty} e^{s_{k}}\right)$.

By (5.2) for every $\varepsilon$ with $0<\varepsilon<1,-b$ one can choose $\bar{a}_{0}$ such that 
$0<\bar{a}_{0}-a_{0}<\log b^{-1}$ (this guarantees the existence of an $\bar{a}_{i}$ satisfying $\left.\sum e^{s_{k}}=1\right)$ and that the right-hand side of (5.9) is greater than $\sum_{k=i}^{\infty} e^{s_{k}}-\varepsilon$ $=1-b-\varepsilon$. Letting $\varepsilon$ tend to zero one gets (5.5).

Summing (5.5) for $i=0,1, \ldots, N$ one has

$$
1 \geqslant \sum_{i=0}^{N} \mu_{i} \geqslant \mu_{0} \sum_{i=0}^{N} \sum_{k=i}^{\infty} e^{s_{k}}
$$

Because of $\sum(k+1) e^{s_{k}}=\infty$ the right-hand side of (5.10) tends to $+\infty(N$ $\rightarrow \infty)$, a contradiction. This proves the theorem.

Finally, the author would like to thank K. Sigmund for encouragement and valuable advice.

\section{REFERENCES}

1. R. Bowen, Equilibrium states and the ergodic theory of Anosov diffeomorphisms, Lecture Notes in Math., vol. 470, Springer-Verlag, Berlin and New York, 1975.

2. B. Felderhof and M. Fisher, four articles, Ann. Phys. 58 (1970), 176, 217, 268, 281.

3. W. K. Feller, An introduction to probability theory and its applications. Vol. I, 2 nd ed., Wiley, New York; Chapman and Hall, London, 1957. MR 19, 466.

4. M. Fisher, Physica 3 (1967), 255-283.

5. F. Ledrappier, Principe variationnel et systemes dynamiques symboliques, $\mathrm{Z}$. Wahrscheinlichkeitstheorie und Verw. Gebiete 30 (1974), 185-202.

6. D. Ruelle, Statistical mechanics on a compact set with $\mathbf{Z}^{\prime}$ action satisfying expansiveness and specification, Trans. Amer. Math. Soc. 185 (1973), 237-252.

7. P. Walters, $A$ variational principle for the pressure of continuous transformations, Amer. J. Math. 97 (1975), 937-971.

8. - Ruelle's operator theorem and g-measures, Trans. Amer. Math. Soc. (to appear).

9. B. Weiss, Intrinsically ergodic systems, Bull. Amer. Math. Soc. 76 (1970), 1266-1269. MR 42 \# 1978.

MATHematisches INSITITUt der Universität, A-1090 Wien, StrudLhofgasSe 4, AUSTRIA 